\title{
Vanin-1 Gene Expression in Acne Vulgaris and its Correlation with Disease Severity
}

\author{
Aya Abd-El-Hamied Amin ${ }^{1}$, Abd-ElAziz El-Rifaie ${ }^{1}$, Laila Ahmed Rashed ${ }^{2}$, Shaimaa Taha Osman ${ }^{1}$ \\ ${ }^{1}$ Dermatology Department, Faculty of Medicine, Beni Suef University, Beni Suef, Egypt. \\ ${ }^{2}$ Biochemistry department, Faculty of Medicine, Cairo University, Cairo, Egypt
}

\begin{abstract}
Background: Acne vulgaris is the most prevalent chronic disease worldwide, occurs during adolescence. The pathogenesis is multifactorial with different grades of severity and the human vanin gene family especially vanin-1 might play a role in pathogenesis of acne. Aim: explore the role of Vanin-1 in acne vulgaris and its correlation with disease severity, duration, weight and residency. Patient and method: 42 acne patients and 42 age and sex matched healthy controls were enrolled. Skin biopsies were taken from the patient and normal skin of controls. Vanin-1 gene expression was estimated by real time PCR. Results: Expression of Vanin-1 gene in acne patients was significantly higher than healthy controls ( $p$-value $<0.001)$, also, there are positive correlation between VNN1 gene expression and patients weight and acne duration $(\mathrm{P}<0.001)$, also VNN1 gene expression is significantly higher among urban as compared with rural patients. Conclusion: VNN1 gene expression is significantly higher in acne patients than healthy controls indicating thatVanin1 gene involved in pathogenesis of acne vulgaris. In dermatological diseases; Vanin-1 might be a new promising target for therapeutic intervention in acne vulgaris.
\end{abstract}

Keywords: Vanin-1 Gene, Acne Vulgaris, dermatology.

\section{Introduction}

Acne vulgaris is the most common chronic pilo-sebaceous unit disease at age of puberty with severity differs among individuals, its prevalence is about $90 \%$ in teenagers [1]. It is classified as non-inflammatory type of closed or open comedons, and inflammatory type of papules and nodules or a mixture of them, most commonly on facial region, chest and back of human [2]. The pathogenesis of acne vulgaris is multifactorial includes; androgen mediated sebum production, keratinous obstruction of sebaceous follicle outlet, bacterial colonization 
with probionibacterium acnes and inflammatory reaction that plays a major role in acne vulgaris progress [3].

The Vanin gene in human found on chromosome 6q22-24 composed of 3 genes including Vanin-1 (VNN1), Vanin-2 (VNN2) and Vanin-3 (VNN3). Vanins identified as group of enzymes implicated in synthesis of coenzyme A [4]. These enzymes convert pantotheine into pantothenic acid (vitamin B5, precursor of coenzyme A) and into the antioxidant cysteamine that have important role in inflammatory and oxidative processes [4]. VNN1 is an important mediator in lipid biosynthesis and was recognized as an obesity associated gene [5].

Vanin gene family previously investigated in other dermatological diseases as psoriasis, atopic dermatitis, icthyosis as well as non dermatological diseases also expression of Vanin gene differs from dermatological diseases themselves and normal skin [6]. More over VNN1 act as an indicator of disease severity or might be therapeutic agent in systemic sclerosis [7], in inflammatory bowel as it control innate immunity [8], in colorectal carcinoma [9], in hypertension (HTN) to regulate blood pressure [10].

From our reviewing literature there were no available studies about the role of vanin gene in acne vulgaris. We proposed that Vanin-1 gene might play role in acne pathogenesis. Therefore the aim of our study is to detect level of Vanin1 in acne vulgaris and its correlation with disease severity, duration, weight and residency.

\section{Patients and Methods}

The study was conducted on 42 patients with acne vulgaris and 42 age, sex matched controls. The study was approved by local ethical committees in the faculty of medicine, Beni Suef University. All study subjects were collected from dermatology clinic faculty of medicine, Beni Suef University in the period between February 2019 and August 2019.

\subsection{Inclusion criteria:}

- Both sexes will be included.

- Age between 16 to 25 years.

- Patients with acne vulgaris.

- Healthy control group will be age and sex matched with our patients.

\subsection{Exclusion criteria:}

- Age $<16$ years and $>25$ years.

- Patients on phototherapy.

- Patients receiving drug interfere with parameter to be estimated.

- Patients with systemic complications.

- Immuno-compromised patients.

- Patients have cutaneous tumors.

- Patients with chronic diseases.

- Patients requiring emergency medical and surgical intervention.

- -Pregnant or lactating females. 


\subsection{Statistical methodology}

Comparison of quantitative variables between the study groups was carried out using the Mann-Whitney U- test for independent samples. Comparison of sex distribution between the study groups was carried out using the $\mathrm{X} 2$ test. The correlation between various variables was assessed using the Spearman rank correlation equation for non normal variables. The significance level was set at $\mathrm{P} \leq 0.05$. All statistical calculations were carried out using Microsoft Excel (2007: Microsoft Corp.,
Redmond, W A, USA) and SPSS (v15.0 for windows; SPSS Inc., Chicago, IL, USA).

\section{Results}

The current study was conducted at Beni-Suef university hospital within six months from February to August 2019. The participants were randomly grouped into two groups, 42 in each group. Group 1: acne patients and group 2: controls. There were no significant differences between patients and controls regarding the age and sex.

Table1.Comparison of the Vanin-1 Gene Expression in Acne Vulgaris and in Controls:

\begin{tabular}{|c|c|c|c|}
\hline & $\begin{array}{l}\text { Cases } \\
\mathrm{N}=42\end{array}$ & $\begin{array}{c}\text { Controls } \\
\mathrm{N}=42\end{array}$ & p-value \\
\hline Mean \pm SD & $3.59 \pm 1.8$ & $1.06 \pm 0.2$ & \multirow[t]{4}{*}{$0.001^{*}$} \\
\hline Range & 1.01 & 0.78 & \\
\hline Minimum & 7.02 & 0.82 & \\
\hline Maximum & 8.03 & 1.60 & \\
\hline
\end{tabular}

*P-value $\leq \mathbf{0 . 0 5}$ is considered significant by (Mann-Whitney U test).

Table (1): show Expression of Vanin-1 Gene in acne patients was significantly higher than in healthy controls ( $p$-value $=0.001)$; where the mean levels were (3.59 vs. 1.06$)$ in cases and controls respectively.

Table2. Correlation between the Vanin-1 gene expression in acne vulgaris and acne grade; $(\mathrm{N}=42)$ :

\begin{tabular}{lcc}
\hline \multicolumn{2}{c}{ Acne Grade } \\
Vanin-1 Gene Expression & $r=-0.119$ & $p$-value $=0.455$ \\
\hline & rSpearman's Rank correlation
\end{tabular}

Table (2): show There were no detected correlation between acne grade and Vanin-1 gene expression in acne vulgaris patients; $p$-value $>0.05$. 


\section{Discussion}

To the best of our knowledge, this is a novel study to be the first that link Vanin-1 gene expression to acne vulgaris. There are positive correlation between VNN1 gene expression (P-value $\leq \mathbf{0 . 0 5})$ and acne patients, disease duration and urban patients. But there are no relation between sex, age, working status, disease course, recurrence history, family history, acne grades and VNN1 gene expression ( $\mathrm{p}$-value $>0.05$ ). The patients were predominantly urban, obese, grade II and IV by Global Acne Severity Scale with progressive course $(61.9 \%)$ and average disease duration was $16.80 \pm 12.6$ months [11].

VNN1 gene expression in acne patients is significantly higher than in healthy controls ( $p$ value $=0.001$ ). It is a novel data and the most important finding that matched our suggestion as VNN1 might be a role player in acne pathogenesis through conversion of pantotheine into pantothenic acid (vitamin B5, a coenzyme A precursor) and into antioxidant cysteamine as inflammation and oxidative stress play a major role in development of acne vulgaris.

Agreement of our data from literature; $\mathrm{CoA}$ is a critical agent important in lipid metabolism and other cellular processes also there is important association between CoA metabolism and inflammation [12].
The pantetheinase enzyme that recycles pantothenic acid and pantetheinase gene (vanin-1) has been shown to be involved in the progression of inflammatory conditions that matched our data that VNN1 has a role in acne vulgaris through increasing inflammation [12].

Propionibacterium acne Promotes Th17 and mixed Th17, Th1 responses in acne vulgaris also $\mathrm{P}$. acnes can induce IL-17 production by $\mathrm{T}$ cells suggests that acne might be a T helper type 17 (Th17) mediated disease [13]. More over combination of Th1 cytokines and Th17 cytokines resulted in a strong synergistic induction of both vanin-1 and vanin-3 expressions, and IL-17 alone strongly induced vanin-1 and vanin-3 expression and that matched our suggestion that links VNN1 with acne pathogenesis [6]. With agreement of our study found that the level of VNN1 gene was higher in psoriasis vulgaris as psoriasis is TH17 mediated disease and it was mediated by increased level of IL-17 and so VNN1 has a role in its pathogenesis [4].

Vanins are pantotheinases andVNN1 act as inflammatory mediator so treatment of acne may be directed through targeting the vanin gene [14].

Pantothenic acid is main component of CoA also deficiency of $\mathrm{CoA}$ result in decreased metabolism and oxidation of fatty acids and skin becomes oily causing acne. In addition CoA is important in hormonal 
synthesis as well as oxidation and metabolism of fatty acid [15]. Also pantothenic acid regulate barrier function of epidermis and differentiation of keratinocytes via $\mathrm{CoA}$ metabolism as $\mathrm{CoA}$ is important in lipid metabolism and other cellular processes so that pantothenic acid suggested as possible new approach in treatment of acne vulgaris [11].

\section{Conclusion and Recommendations}

VNN1 gene expression is significantly higher in acne patients than in health control so VNN1might be a new promising target for therapeutic intervention in acne vulgaris through control of inflammation and oxidative stress. The study recommends further investigations of vanin genes particularly VNN1 and its relation to acne treatment

\section{References}

1. Mustafa AI, El-Shimi OS. Serum irisin: A prognostic marker for severe acne vulgaris. Journal of cosmetic dermatology, 2018; 17(5):931-34.

2. Qidwai A, Pandey M, Pathak S, Kumar R, Dikshit A. The emerging principles for acne biogenesis: A dermatological problem of puberty. Human Microbiome. J. 2017; 4: 713.

3. Jiang H, Li C. Common Pathogenesis of Acne Vulgaris and Atherosclerosis. Inflammation, 2018; 1-5.
4. Doss RW, Rashed LA, Emad El-Din Arafa A. Association between VNN1 gene polymorphism and the risk for psoriasis. $J$ of the Egyptian Women's Dermatologic Society, 2018; 15(2): 106-10.

5. Jacobo-Albavera L, Aguayo-de la Rosa PI, Villarreal-Molina, T, Villamil-Ramírez H, León-Mimila $\quad \mathrm{P}, \quad$ Romero-Hidalgo $\mathrm{S}$, Aguilar-Salinas CA. VNN1 gene expression levels and the G-137T polymorphism are associated with HDL-C levels in Mexican prepubertal children. PloS one, 2012; 7(11): e49818.

6. Jansen PAM, Kamsteeg M, Olthuis DR, van Vlijmen IMJ, de JonghGH, Bergers M, et al. Expression of the vanin gene family in normal and inflamed human skin: induction by proinflammatory cytokines. $J$ Invest Dermatol. 2009; 129: 2167-74.

7. Kavian N, Mehlal S, Marut W, Servettaz A, Giessner C, Bourges C, et al. Imbalance of vanin pathway in systemic sclerosis. $J$ Immunol. 2016; 197:3326-35.

8. Naquet P, Pitari G, Dupre S, Galland F. Role of the Vnn1 pantetheinase in tissue tolerance to stress. Biochem Soc Trans. 2014; 42(4):1094-100.

9. Surya R, Héliès-Toussaint C, Martin OC, Gauthier T, Guéraud F, Taché S, Joly C. Red meat and colorectal cancer: Nrf2dependent antioxidant response contributes to the resistance of preneoplastic colon cells 
to fecal water of hemoglobin-and beef-fed rats. Carcinogenesis, 2016; 37(6): 635-45.

10. Kaskow BJ, Diepeveen LA, Michael Proffitt J, Rea AJ, Ulgiati D, et al. Molecular prioritization strategies to identify functional genetic variants in the cardiovascular disease associated expression QTL Vanin-1. Eur J Hum Genet. 2014; 22: 688-95.

11. Dreno B, Poli F, Pawin H, Beylot C, Faure M, Chivot M, Revuz, J. Development and evaluation of a Global Acne Severity scale (GEA scale) suitable for France and Europe. Journal of the European Academy of Dermatology and Venereology, 2011; 25(1): 43-8.

12. Yang M, Moclair B, Hatcher V, Kaminetsky J, Mekas M, Chapas A, Capodice J. A randomized, double-blind, placebo-controlled study of a novel pantothenic acid-based dietary supplement in subjects with mild to moderate facial acne. Dermatology and therapy, 2014; 4(1): 93-101.

13. Kistowska M, Meier B, Proust T, Feldmeyer L, Cozzio A, Kuendig T, French LE. Propionibacterium acnes promote Th17 and Th17/Th1 responses in acne patients. $J$ of Investigative Dermatology, 2015; 135(1): 110-18.

14. Van Diepen JA, Jansen PA, Ballak DB, Hijmans A, Rutjes FP, Tack CJ, Stienstra
R. Genetic and pharmacological inhibition of vanin-1 activity in animal models of type 2 diabetes. Scientific reports, 2016; 6: 21906.

15. Fan L, Cai Z, Zhang K, Han F, Li J, He C, Wang H. Green electrospun pantothenic acid/silk fibroin composite nanofibers: Fabrication, characterization and biological activity. Colloids and surfaces b. Biointerfaces, 2014; 117: 14-20. 